msh-mss Mathématiques et sciences humaines

181 | Printemps 2008

Varia

\title{
Projective operations on relational constraints
}

Opérations projectives sur contraintes relationnelles

\section{Luigi Burigana}

\section{OpenEdition}

Journals

Édition électronique

URL : https://journals.openedition.org/msh/8923

DOI : $10.4000 /$ msh. 8923

ISSN : 1950-6821

\section{Éditeur}

Centre d'analyse et de mathématique sociales de l'EHESS

\section{Édition imprimée}

Date de publication : 20 mars 2008

Pagination : 35-58

ISSN : 0987-6936

\section{Référence électronique}

Luigi Burigana, "Projective operations on relational constraints », Mathématiques et sciences humaines [En ligne], 181 | Printemps 2008, mis en ligne le 27 mars 2008, consulté le 21 septembre 2021. URL : http://journals.openedition.org/msh/8923 ; DOI : https://doi.org/10.4000/msh.8923 


\title{
PROJECTIVE OPERATIONS ON RELATIONAL CONSTRAINTS
}

\author{
Luigi BURIGANA ${ }^{1}$
}

\begin{abstract}
RÉSUMÉ - Opérations projectives sur contraintes relationnelles Étant donné un ensemble de variables et un ensemble de valeurs, par contrainte (relationnelle), nous entendons tout ensemble de fonctions du premier ensemble vers le deuxième ensemble. Sont ici considérées deux opérations spéciales sur les contraintes, appelées projection existentielle et projection universelle en raison de leur similitude avec les quantificateurs existentiel et universel dans un calcul prédicatif. On explore le pouvoir expressif des deux opérations, c'est-à-dire, les propriétés générales de la variété de contraintes qui peuvent être produites à partir de quelques contraintes initiales et en appliquant ces opérations une ou plusieurs fois. Sont également présentés quelques commentaires en ce qui concerne le pouvoir expressif d'un système plus large, comprenant les opérations projectives et booléennes (i.e., complémentation, union et intersection) sur les contraintes.

MOTS CLÉS - Contrainte, Pouvoir expressif, Projection, Relation

SUMMARY - Given a set of variables and a set of values, by a (relational) constraint we mean any set of functions from the former to the latter. Two special operations on constraints are considered, called existential and universal projections, because of their similarity with existential and universal quantifiers in a predicate calculus. The expressive power of both operations is explored, i.e., the general properties of the variety of constraints which may be produced starting from some initial constraint and applying those operations one or more times. A few comments are added concerning the expressive power of a larger system, comprising projective and Boolean operations (i.e., complementation, union and intersection) on constraints.
\end{abstract}

KEYWORDS - Constraint, Expressive power, Projection, Relation

\section{INTRODUCTION}

Our analysis refers to a context formed of two basic sets: a set $W$ of variables and a set $O$ of values admissible for those variables. Both sets are presumed to be of finite cardinality. The meanings of "variable" and "value" may vary widely in differing applications of the paradigm to be discussed. For example, if we consider the variety of pictures obtainable by colouring the cells of a matrix of fixed size, then the variables may be identified with the cells in the matrix and the values with the colours available for painting. If we plan activities during a certain period of time, which is divided into a fixed series of sub-periods, then those sub-periods may

\footnotetext{
${ }^{1}$ Dipartimento di Psicologia Generale, Università di Padova, Via Venezia 8, I-35131 Padova, Italy, luigi.burigana@unipd.it
} 
be taken as the variables, and the various activities to be performed as the values admissible for the variables. If the coach of a soccer team wants to define the line-up for a match, having a certain number of players at his disposal, then each of the eleven positions to be filled in is a variable, and the available players are the values.

By a (global) value assignment in context $(W, O)$, we mean any function from $W$ to $O$, and use symbol $O[W]$ to denote the set of all such assignments - in ordinary notation, this is $O^{W}$. A relational constraint (or, more simply, a constraint) in context $(W, O)$ is specified as a set of (global) assignments, i.e., a subset of $O[W]$. In general, a constraint involving all variables in the context originates as follows. First, a definite subset $U \subseteq W$ of variables is taken into consideration, on which a certain local constraint $C$ is determined as a subset of $O[U]$ — this is the set of all functions from $U$ to $O$. Then a constraint $A$ on the whole set of variables $W$ is derived, as the global expansion of local constraint $C$, i.e.:

$$
A=\{p+q: p \in C, q \in O[W \backslash U]\}
$$

In this equation, term $p+q$ (called the catenation of $p$ and $q$ ) amounts to the settheoretic union of $p$ and $q$, which are functions - thus, sets of ordered pairs - on disjoint domains $U$ and $W \backslash U$ (more generally, for any $U, V \subseteq W$, if $p \in O[U]$, $q \in O[V]$ and $U \cap V=\emptyset$, then $p+q \in O[U \cup V])$.

To illustrate this point, let us consider the case of a soccer coach who is studying the plausible formation of his team for a match. In this case, $W=\left\{w_{1}, \ldots, w_{11}\right\}$ is the regular set of positions in the team, $O=\left\{o_{1}, \ldots, o_{n}\right\}$ is the set of players at the coach's disposal (with $n \geq 11$ ). Let us assume that he begins his planning by considering set $U=\left\{w_{7}, w_{8}, w_{9}, w_{10}, w_{11}\right\}$ of the forward positions in the team. An assignment of players to these positions is an (injective) function $\left\{\left(w_{7}, o\left(w_{7}\right)\right), \ldots,\left(w_{11}, o\left(w_{11}\right)\right)\right\}$ from $U$ into $O$, the plausibility of which depends not only on the adequacy of each single player to his assigned position, but also on the consistency between players who play together (and on the foreseeable line-up of the opposing team). Through his analysis, the coach identifies a set of alternative plausible assignments of players to the forward positions, i.e., a subset $C$ of $O[U]=O\left[\left\{w_{7}, \ldots, w_{11}\right\}\right]$, which is a local constraint on variables $w_{7}, \ldots, w_{11}$. The global expansion of $C$, specified by (1), is the set of all complete assignments of players to the eleven positions such that their portions concerning positions $\left\{w_{7}, \ldots, w_{11}\right\}$ are among the local assignments accepted in $C$. This is a global constraint, i.e., a subset of $O[W]=O\left[\left\{w_{1}, \ldots, w_{11}\right\}\right]$. Pursuing his analysis, the coach may then consider other special sections of the team, such as set $\left\{w_{1}, w_{2}, w_{3}\right\}$ of defence positions, set $\left\{w_{2}, w_{4}, w_{7}, w_{8}\right\}$ of right-hand positions, etc., and for each section specify a set of plausible assignments. In this way he creates a "network of constraints" (in the sense of [Montanari, 1974; Dechter, 2003]), which will serve as a guide for identifying a plausible, possibly optimal formation of the soccer team as a whole.

In this study, we examine two special operations on constraints, called "existential projection" and "universal projection" (the reason for the adjectives in these titles is that there is a natural correspondence between both projective operations and existential and universal quantifiers in a first-order predicate calculus, as will appear in the definitions introduced in Section 2). Both projective operations form 
a fragment of a set-theoretic calculus on relational constraints (other basic components of the calculus, corresponding to Boolean operations on sets, will be briefly considered in Section 6). More precisely, existential projection is explicitly specified in any standard presentation of the theory of constraint networks, where it is simply called "projection" and is usually written as $\pi_{V}(A)$, terms $A$ and $V$ being a constraint and a set of variables with respect to which the projection is taken (note that $\pi_{V}(A)$ equals $A \uparrow(W \backslash V)$ as defined by (2) in the next section; cf. [Dechter, 2003, §1.3]). The same concept, called by the same name and denoted by the same symbol, also occurs among the primitives of the theory of relational databases, which is akin to the theory of constraint networks in various respects (cf. [Maier, 1983, §2.3]). Instead, what we call "universal projection" is not found among the primitives of these theories. However, it may be directly expressed in terms of two primitives of the theories - i.e., projection and complementation - through a scheme which parallels the equivalence of formulas $(\forall x)(p(x))$ and $\neg(\exists x)(\neg p(x))$ in predicate logic.

My specific purpose with this study is to analyse the "expressive power" of projective operations on constraints, which means exploring the variety of constraints which may be derived from some given constraints by applying to them existential and/or universal projections, an arbitrary finite number of times (on the concept of "expressive power" as referred to a system of basic constraints, cf. [Jeavons, Cohen, Gyssens, 1999]). As we soon see, both existential and universal projections involve two factors, an input constraint $A$ and a set of variables $U$. Of these two factors, it is the second one which plays the most active part in the analyses to be presented in this paper. On one hand, we explore the inner organization of the variety of constraints which may be obtained by keeping input constraint $A$ fixed and applying only once either an existential or a universal projection relative to a set $U$, which varies in the universe of all subsets of set $W$ of variables in the context. On the other hand, we search for a reduced variety of regular finite sequences of existential and universal projections which is fully representative of the variety of all constraints obtainable from one fixed input constraint through arbitrary finite sequences of projections (of the existential and/or universal type) and in relation to arbitrary sets of variables. In the concluding part of the paper we argue that these targets for analysis may be viewed as problems of "definability theory", as specifically applied to relational constraints, and make a few references showing the importance of such problems for formal developments in psychological science, and social sciences more generally.

In detail, after defining the two projective operations and making explicit a few of their elementary properties (Section 2), we introduce the concepts of free and closed sets of variables, relative to a given constraint (Section 3). Then the expressive power of projective operations is discussed, first by considering one single application of the one or the other operation (Section 4), and then by referring to arbitrary finite sequences of applications (Section 5). We then add some notes regarding the combined expressive power of projective and Boolean operations on constraints (Section 6) and conclude by comparing the subject of our analysis with concepts in other mathematical fields, and by remarking upon the main results of this study (Section 7). 


\section{EXISTENTIAL AND UNIVERSAL PROJECTIONS}

Let $A \subseteq O[W]$ be any constraint and $U \subseteq W$ any set of variables. The e-projection (existential projection) of $A$ relative to $U$, denoted by $A \uparrow U$, is the set containing each assignment in context $(W, O)$ such that there is a way of modifying its subassignment on $U$ so that the resulting assignment is in $A$. The u-projection (universal projection) of $A$ relative to $U$, denoted by $A \downarrow U$, is the set containing each assignment in context $(W, O)$ such that for every modification of its sub-assignment on $U$, the resulting assignment is in $A$. The two concepts are formally rendered by these equations:

$$
\begin{aligned}
& A \uparrow U=\left\{p+q: p^{\prime}+q \in A \text { for some } p^{\prime} \in O[U]\right\} \\
& A \downarrow U=\left\{p+q: p^{\prime}+q \in A \text { for all } p^{\prime} \in O[U]\right\}
\end{aligned}
$$

in which + is catenation between local assignments and $q$ stands for a generic member of $O[W \backslash U]$.

The following two inclusions are directly implied by definitions (2) and (3):

$$
A \downarrow U \subseteq A \subseteq A \uparrow U
$$

Also consider this equivalence:

$$
A \downarrow U=A \text { if and only if } A \uparrow U=A \text {. }
$$

To prove it, first assume $A \downarrow U=A$. For any $s$, if $s \in A \uparrow U$, then $s=p+q$ and $p^{\prime}+q \in A$ for some $p, p^{\prime} \in O[U]$ and $q \in O[W \backslash U]$, so that $p^{\prime}+q \in A \downarrow U$ due to hypothesis $A=A \downarrow U$, whence $s=p+q \in A$ because of (3). This proves inclusion $A \uparrow U \subseteq A$, and also equality $A \uparrow U=A$, since the reciprocal inclusion is ensured by (4). The converse implication is verified in a similar way.

The result of a projection depends on both factors $A$ and $U$, the former being any subset of $O[W]$ (including extremes $A=\emptyset$ and $A=O[W]$ ) and the latter any subset of $W$ (including extremes $U=\emptyset$ and $U=W$ ). The results for extreme choices of factors are given by the following rules:

$$
\begin{aligned}
& A \uparrow \emptyset=A=A \downarrow \emptyset \\
& A \uparrow W=O[W] \text { or }=\emptyset \text { depending on whether } A \neq \text { or }=\emptyset \\
& A \downarrow W=O[W] \text { or }=\emptyset \text { depending on whether } A=\text { or } \neq O[W] .
\end{aligned}
$$

To verify the equations in the first line, consider that $O[\emptyset]=\{\sharp\}$ where $\sharp$ is the null assignment (i.e., the empty set of pairs of elements in $W$ and elements in $O$ ) and that $p+\sharp=p=\sharp+p$ for all $p \in O[U]$ and $U \subseteq W$ (i.e., the null assignment is the identity for catenation). The rules in the second and third lines are obvious.

The next proposition is a list of elementary properties of projections, involving relations or operations on their factors.

Proposition 1. For all $A, B \subseteq O[W]$ and $U, V \subseteq W$ :

(i) if $A \subseteq B$, then $A \uparrow U \subseteq B \uparrow U$ and $A \downarrow U \subseteq B \downarrow U$; 
(ii) $(A \uparrow U)^{c}=A^{c} \downarrow U$ and $(A \downarrow U)^{c}=A^{c} \uparrow U$;

(iii) if $U \subseteq V$, then $A \uparrow U \subseteq A \uparrow V$ and $A \downarrow U \supseteq A \downarrow V$;

(iv) $(A \uparrow U) \uparrow V=A \uparrow U \cup V$ and $(A \downarrow U) \downarrow V=A \downarrow U \cup V$.

Proof. Part (i) is obvious. To prove part (ii), let $p$ and $q$ denote generic (local) assignments in $O[U]$ and $O[W \backslash U]$, respectively. Then $p+q \in(A \uparrow U)^{c}$ iff $p^{\prime}+q \in A$ for no $p^{\prime} \in O[U]$ iff $p^{\prime}+q \in A^{c}$ for all $p^{\prime} \in O[U]$ iff $p+q \in A^{c} \downarrow U$, giving the first equality. The second equality is obtained in a similar way. To prove part (iii), let $p$, $q$ and $r$ denote generic assignments in $O[U], O[V \backslash U]$ and $O[W \backslash V]$, respectively, so that $p+q+r \in O[W]$ if $U \subseteq V$. Now, if $p+q+r \in A \uparrow U$, then $p^{\prime}+q+r \in A$ for some $p^{\prime} \in O[U]$, thus also $p^{\prime}+q^{\prime}+r \in A$ for some $p^{\prime}+q^{\prime} \in O[V]$ (put $q^{\prime}=q$ ), whence $p+q+r \in A \uparrow V$, which implies the first inclusion. Similarly, if $p+q+r \in A \downarrow V$, then $p^{\prime}+q^{\prime}+r \in A$ for all $p^{\prime}+q^{\prime} \in O[V]$, so that $p^{\prime}+q+r \in A$ for all $p^{\prime} \in O[U]$, whence $p+q+r \in A \downarrow U$, which gives the second inclusion. To verify part (iv), let us denote by $p, q, r$ and $s$ generic assignments in $O[U \backslash V], O[U \cap V], O[V \backslash U]$ and $O[W \backslash(U \cup V)]$, respectively. Then consider the following chain of double implications, which are directly ensured by definitions (2) and (3):

$$
\begin{aligned}
& p+q+r+s \in(A \uparrow U) \uparrow V \text { iff } \\
& \text { there is } q^{\prime}+r^{\prime} \in O[V], \text { so that } p+q^{\prime}+r^{\prime}+s \in A \uparrow U \text { iff } \\
& \text { there is } q^{\prime}+r^{\prime} \in O[V], \\
& \text { so that (there is } \left.p^{\prime}+q^{\prime \prime} \in O[U] \text {, so that } p^{\prime}+q^{\prime \prime}+r^{\prime}+s \in A\right) \text { iff } \\
& \text { there is } p^{\prime}+q^{\prime \prime}+r^{\prime} \in O[U \cup V] \text {, so that } p^{\prime}+q^{\prime \prime}+r^{\prime}+s \in A \text { iff } \\
& p+q+r+s \in A \uparrow U \cup V .
\end{aligned}
$$

This proves the first equation in part (iv). The second equation is directly implied by the first, the second equation in (ii), and the involutive property of complementation.

\section{FREE SETS AND CLOSED SETS OF VARIABLES}

Let $A \subseteq O[W]$ be any constraint and $U \subseteq W$ any set of variables. We say that $U$ is free under $A$ if equality $A \uparrow U=A$ holds true (which is equivalent to $A \downarrow U=A$, in view of $(5))$. In other words:

$$
\begin{aligned}
& U \text { is free under } A \text { iff } \\
& p+q \in A \text { implies } p^{\prime}+q \in A, \text { for all } p, p^{\prime} \in O[U], q \in O[W \backslash U] .
\end{aligned}
$$

Some elementary properties of the concept are directly apparent, based on results in the previous section. It is seen, for example, that (4) implies that every set of variables is free under both the empty constraint $A=\emptyset$ and the largest constraint $A=O[W]$, and that (6) implies that the empty set of variables $U=\emptyset$ is free under every constraint.

Other useful properties of the concept are the following. 
Proposition 2. For any constraint $A \subseteq O[W]$ and all sets of variables $U, V \subseteq W$ :

(i) if $U \subseteq V$ and $V$ is free under $A$, then so is $U$;

(ii) if both $U$ and $V$ are free under $A$, then so is $U \cup V$;

(iii) set $U$ is free under both $A \uparrow U$ and $A \downarrow U$;

(iv) if $U$ is free under $A$, then it is free under both $A \uparrow V$ and $A \downarrow V$.

Proof. Part (i) follows from (4) and Proposition 1.(iii). Part (ii) is directly implied by Proposition 1.(iv). Part (iii) also follows from Proposition 1.(iv), due to the idempotence of union. Part (iv) is obtained by noting that, if $U$ is free under $A$, then $(A \uparrow V) \uparrow U=A \uparrow(U \cup V)=(A \uparrow U) \uparrow V=A \uparrow V$ and, similarly, $(A \downarrow V) \downarrow U=A \downarrow V$, due to Proposition 1.(iv).

Some more properties are collected in the next proposition. They involve Boolean operations on constraints.

Proposition 3. For all constraints $A, B \subseteq O[W]$ and any set of variables $U \subseteq W$ : (i) if $U$ is free under $A$, then $A \cup(B \uparrow U)=(A \cup B) \uparrow U, A \cap(B \uparrow U)=(A \cap B) \uparrow U$, $A \cup(B \downarrow U)=(A \cup B) \downarrow U$ and $A \cap(B \downarrow U)=(A \cap B) \downarrow U$;

(ii) if $U$ is free under $A$, then it is also free under $A^{c}$, and if it is free under both $A$ and $B$, then it is also free under both $A \cup B$ and $A \cap B$.

Proof. To prove the first equation in part (i), let us suppose that $U$ is free under $A$ and use letters $p$ and $q$ to denote assignments in $O[U]$ and $O[W \backslash U]$, respectively. Then the equation is accounted for by the following chain of double implications:

$$
\begin{aligned}
& p+q \in A \cup(B \uparrow U) \text { iff } \\
& p+q \in A \text { or } p+q \in B \uparrow U \text { iff } \\
& p+q \in A \text { or }\left(p^{\prime}+q \in B \text { for some } p^{\prime} \in O[U]\right) \text { iff } \\
& \left(p^{\prime}+q \in A \text { or } p^{\prime}+q \in B\right) \text { for some } p^{\prime} \in O[U] \text { iff } \\
& p^{\prime}+q \in A \cup B \text { for some } p^{\prime} \in O[U] \text { iff } \\
& p+q \in(A \cup B) \uparrow U .
\end{aligned}
$$

The only point in this chain which deserves comment is part "if" between the third and fourth lines. Suppose that the condition in the fourth line is true. If $p^{\prime}+q \in B$, then the second disjunct in the third line is obviously true. If $p^{\prime}+q \in A$, then the first disjunct in the third line is true, due to the hypothesis that $U$ is free under $A$. The second equation in part (i) is proven by a chain of double implications obtained from the previous one by replacing intersection $\cap$ for union $\cup$, and conjunction "and" for disjunction "or". The third and fourth equations are derived from the first two by applying Proposition 1.(ii), the involutive property of complementation and De Morgan laws. As for part (ii), note that the first property in it is a corollary of Proposition 1.(ii), whereas the second and third properties are implied by equations in part (i) of this proposition. In detail, if $U$ is free under both $A$ and $B$, then $(A \cup B) \uparrow U=A \cup(B \uparrow U)=A \cup B$ and $(A \cap B) \uparrow U=A \cap(B \uparrow U)=A \cap B$.

Parts (i) and (ii) in Proposition 2 mean that, for any constraint $A$, the set of all sets of variables which are free under it is an ideal in the field of all subsets of 
$W$ (the power set $2^{W}$ as a Boolean algebra) $)^{2}$. Thus, the largest free set of variables under $A$ does exist. It is denoted by $F(A)$ and defined as:

$$
F(A)=\bigcup\{U \subseteq W: U \text { is free under } A\} .
$$

When referred to all constraints $A \subseteq O[W]$, this equation gives rise to a mapping $F$ from $2^{O[W]}$ to $2^{W}$. Some properties of mapping $F$ are easily recognised. For example, from (4) we deduce that $F(A)=W$ if and only if either $A=O[W]$ or $A=\emptyset$ (the opposite case, $F(A)=\emptyset$, qualifies $A$ as an "irreducible" constraint). Thus $F(O[W])=F(\emptyset)$ and, more generally, $F(A)=F\left(A^{c}\right)$ for all $A \subseteq O[W]$, due to the first statement in Proposition 3.(ii). Inclusions $U \subseteq F(A \uparrow U), U \subseteq F(A \downarrow U)$, $F(A) \subseteq F(A \uparrow U)$ and $F(A) \subseteq F(A \downarrow U)$ are true in general, owing to parts (iii) and (iv) of Proposition 2.

Now, suppose that any constraint $A$ is fixed and that, for each set of variables $U \subseteq W$, the largest free sets under e-projection $A \uparrow U$ and u-projection $A \downarrow U$ are considered, which we denote by $\epsilon_{A}(U)$ and $v_{A}(U)$, so that:

$$
\begin{aligned}
& \epsilon_{A}(U)=F(A \uparrow U) \\
& v_{A}(U)=F(A \downarrow U) .
\end{aligned}
$$

By so doing, two mappings $\epsilon_{A}$ and $v_{A}$ from $2^{W}$ into itself are generated. The following proposition sheds light on the general formal profile of these mappings.

PROposition 4. For any constraint $A \subseteq O[W]$, both $\epsilon_{A}$ and $v_{A}$ are closure operators in the power set $2^{W}$ partially ordered by set inclusion.

Proof. Arguments concerning the one and the other mappings are dual to each other, so that we may limit ourselves to discussing one of them, i.e., mapping $\epsilon_{A}$. To conclude that it is a closure operator, we have to check the following conditions, which are referred to arbitrary $U, V \subseteq W:$ (i) $U \subseteq \epsilon_{A}(U)$; (ii) if $U \subseteq V$, then $\epsilon_{A}(U) \subseteq \epsilon_{A}(V)$; (iii) $\epsilon_{A}\left(\epsilon_{A}(U)\right)=\epsilon_{A}(U)$. Condition (i) is ensured by Proposition 2.(iii). To prove condition (ii), let us suppose $U \subseteq V$; if $T \subseteq W$ is free under $A \uparrow U$, then $T$ is also free under $(A \uparrow U) \uparrow V=A \uparrow U \cup V=A \uparrow V$, due to Propositions 1.(iv) and 2.(iv); thus, if $T \subseteq \epsilon_{A}(U)$, then also $T \subseteq \epsilon_{A}(V)$, which implies $\epsilon_{A}(U) \subseteq \epsilon_{A}(V)$. As for condition (iii), we prove it by verifying the following stronger equation:

$$
A \uparrow F(A \uparrow U)=A \uparrow U .
$$

Inclusion from right to left is simply due to inclusion $U \subseteq F(A \uparrow U)$ and Proposition 1.(iii). To prove inclusion from left to right, let us consider any $p \in O[F(A \uparrow U)]$ and $q \in O[W \backslash F(A \uparrow U)]$ such that $p+q \in A \uparrow F(A \uparrow U)$; this means that there is $p^{\prime} \in O[F(A \uparrow U)]$ so that $p^{\prime}+q \in A$, and also $p^{\prime}+q \in A \uparrow U$ due to (4); this last result, combined with the fact that $F(A \uparrow U)$ is a set of variables free under $A \uparrow U$, implies $p+q \in A \uparrow U$. Through generalisation, the required inclusion is obtained.

\footnotetext{
${ }^{2}$ I use the name "field of sets" in accordance with [Halmos, 1963, §3].
} 
Note that, although they are related to the same constraint $A$, operators $\epsilon_{A}$ and $v_{A}$ may be different from each other. A simple example to this effect is as follows. Consider set $A=\{111,101,011\}$, to be interpreted as a constraint in context $(W, O)=\left(\left\{w_{1}, w_{2}, w_{3}\right\},\{0,1\}\right)$ (string 101 signifies assignment $\left\{\left(w_{1}, 1\right),\left(w_{2}, 0\right),\left(w_{3}, 1\right)\right\}$, etc. $)$. It is seen that $A \uparrow\left\{w_{1}\right\}=\{111,101,011,001\}$ and $A \downarrow\left\{w_{1}\right\}=\{111,011\}$, so that $\epsilon_{A}\left(\left\{w_{1}\right\}\right)=\left\{w_{1}, w_{2}\right\} \neq\left\{w_{1}\right\}=v_{A}\left(\left\{w_{1}\right\}\right)$. Note, however, the following general equation, which is due to the first statement in Proposition 3.(ii):

$$
\epsilon_{A}(U)=v_{A^{c}}(U) \text {, for all } U \subseteq W, A \subseteq O[W] .
$$

It counts as one further sign of the duality relationship holding between both closure operators.

Again, let us suppose that some constraint $A$ is fixed. For any set of variables $U \subseteq W$, we say that $U$ is e-closed under $A$ if $U=F(A \uparrow U)$ and that $U$ is $u$-closed under $A$ if $U=F(A \downarrow U)$. Symbols $\mathcal{E}_{A}$ and $\mathcal{U}_{A}$ denote the classes of all sets of variables that prove to be e-closed and, respectively, u-closed under constraint $A$. In other words, $\mathcal{E}_{A}$ and $\mathcal{U}_{A}$ are the ranges of both closure operators in Proposition 4 , i.e.:

$$
\mathcal{E}_{A}=\epsilon_{A}\left(2^{W}\right), \quad \mathcal{U}_{A}=v_{A}\left(2^{W}\right)
$$

Due to a general result in abstract algebra (cf. [Birkhoff, 1967, §5.1]), classes $\mathcal{E}_{A}$ and $\mathcal{U}_{A}$ qualify as lattices when partially ordered by set inclusion. The maximum in both lattices is $W$ (the general set of variables), the minimum is $F(A)$ (the largest set of variables free under $A$ ), and the meet operation is simply set intersection.

\section{THE EXPRESSIVE POWER OF ONE PROJECTION}

Consider arbitrary $A, B \subseteq O[W]$. Constraint $B$ is said to be e-expressible (resp., $u$ expressible) based on $A$ if it can be obtained as the result of one existential projection (resp., universal projection) applied to $A$, i.e., if $B=A \uparrow U$ (resp., $B=A \downarrow U$ ) for some $U \subseteq W$. Symbols $E_{e}(A)$ and $E_{u}(A)$ are used for classes of all constraints that are e-expressible and, respectively, u-expressible on the basis of constraint $A$. Once factor $A$ is fixed, projections $A \uparrow U$ and $A \downarrow U$ for varying factor $U$ describe two mappings from $2^{W}$ into $2^{O[W]}$. Classes $E_{e}(A)$ and $E_{u}(A)$ are the ranges of these mappings.

The next proposition guides us in discovering the structural properties of both classes, by revealing their correspondences with previously examined structures.

Proposition 5. Let $A \subseteq O[W]$ be any constraint and $\epsilon_{A}, v_{A}$ the closure operators it induces on $2^{W}$.

(i) For all $U, V \subseteq W, A \uparrow U=A \uparrow V$ if and only if $\epsilon_{A}(U)=\epsilon_{A}(V)$. Moreover, if $U, V$ are e-closed under $A$, then $A \uparrow U \subseteq A \uparrow V$ if and only if $U \subseteq V$.

(ii) For all $U, V \subseteq W, A \downarrow U=A \downarrow V$ if and only if $v_{A}(U)=v_{A}(V)$. Moreover, if $U, V$ are $u$-closed under $A$, then $A \downarrow U \subseteq A \downarrow V$ if and only if $U \supseteq V$.

Proof. The first statement in part (i) is due to equation (8). Part "if" in the second statement is due to Proposition 1.(iii). In view of this, to prove part "only if" in the 
statement, we may instead verify the following implication:

$$
\begin{aligned}
& \text { if } U, V \text { are e-closed under } A \text { and neither } U \subseteq V \text { nor } V \subseteq U \text {, } \\
& \text { then neither } A \uparrow U \subseteq A \uparrow V \text { nor } A \uparrow V \subseteq A \uparrow U .
\end{aligned}
$$

Let us suppose that $U, V$ are e-closed under $A$ and use letters $p, q$ and $r$ for generic assignments in $O[U \backslash V], O[V]$ and $O[W \backslash(U \cup V)]$, respectively. If not $U \subseteq V$, i.e., $U \backslash V \neq \emptyset$, then $U \backslash V$ is not free under $A \uparrow V$ (being e-closed, $V$ is the greatest free set of variables under $A \uparrow V)$, so that there are $p, p^{\prime} \in O[U \backslash V], q \in O[V]$ and $r \in O[W \backslash(U \cup V)]$, such that $p+q+r \in A \uparrow V$ but $p^{\prime}+q+r \notin A \uparrow V$. Of these two results, the first implies:

$$
p+q^{\prime}+r \in A \text { for some } q^{\prime} \in O[V],
$$

whereas the second means that:

$$
p^{\prime}+q^{\prime \prime}+r \notin A \text { for all } q^{\prime \prime} \in O[V] \text {. }
$$

Now let us consider assignment $s=p^{\prime}+q^{\prime}+r$. Due to (9) we obtain $s \in A \uparrow U$ but, due to (10), we obtain $s \notin A \uparrow V$. Thus, $s \in(A \uparrow U) \backslash(A \uparrow V)$, which implies not $A \uparrow U \subseteq A \uparrow V$. In a similar way, it is proven that, if not $V \subseteq U$, then not $A \uparrow V \subseteq A \uparrow U$ either. Proof of part (i) is thus complete. Part (ii) is dual and may be verified by similar reasoning (note, in particular, that implication " $A \downarrow U \subseteq A \downarrow V$ only if $U \supseteq V$ " is in accordance with Proposition 1.(iii)).

Part (i) of Proposition 5 means that, for any fixed constraint $A$, poset $\left(E_{e}(A), \subseteq\right)$ (i.e., the set of all constraints e-expressible based on $A$ and partially ordered by set inclusion) is isomorphic to poset $\left(\mathcal{E}_{A}, \subseteq\right.$ ) (i.e., the set of all sets of variables e-closed under $A$ and partially ordered by set inclusion). The natural isomorphism from the latter to the former poset is determined by associating with any set of variables $U$ in $\mathcal{E}_{A}$ the existential projection $A \uparrow U$, which is a constraint in $E_{e}(A)$. We know from Section 3 that poset $\mathcal{E}_{A}$ is a lattice, so that, due to isomorphism, poset $E_{e}(A)$ is itself a lattice. The maximum in this lattice is $O[W]=A \uparrow W$, i.e., the largest constraint, when $A \neq \emptyset$ (for $A=\emptyset$ the maximum is $\emptyset$, the empty constraint); the minimum in the lattice is $A=A \uparrow F(A \uparrow \emptyset)=A \uparrow \emptyset$, i.e., the constraint presumed as given. Concerning join and meet operations in the lattice, the following rules hold true for all constraints $B=A \uparrow U$ and $C=A \uparrow V$ in $E_{e}(A)$ :

$$
\begin{aligned}
& B \vee C=A \uparrow U \cup V \\
& B \wedge C=A \uparrow U \cap V, \text { if } U \text { and } V \text { are e-closed under } A .
\end{aligned}
$$

In a dual way, part (ii) of Proposition 5 means that, for any fixed constraint $A$, poset $\left(E_{u}(A), \subseteq\right)$ of constraints u-expressible based on $A$ is dually isomorphic to poset $\left(\mathcal{U}_{A}, \subseteq\right)$ of sets of variables u-closed under $A$. The natural dual isomorphism from the latter to the former poset is determined by associating with any set of variables $U$ in $\mathcal{U}_{A}$ the universal projection $A \downarrow U$, which is a constraint in $E_{u}(A)$. From this relationship and the fact that poset $\mathcal{U}_{A}$ is a lattice, we see that poset $E_{u}(A)$ is itself a lattice. The maximum in it is $A=A \downarrow F(A \downarrow \emptyset)=A \downarrow \emptyset$, i.e., the constraint presumed as given; the minimum is the empty constraint $\emptyset=A \downarrow W$ 
when $A \neq O[W]$ (the minimum is $O[W]$ if $A=O[W]$ ). Concerning join and meet operations in the lattice, the following rules hold true for all constraints $B=A \downarrow U$ and $C=A \downarrow V$ in $E_{u}(A)$ :

$$
\begin{aligned}
& B \vee C=A \downarrow U \cap V, \text { if } U \text { and } V \text { are u-closed under } A \\
& B \wedge C=A \downarrow U \cup V .
\end{aligned}
$$

Note that, for any constraint $A$ and its complement $A^{c}=O[W] \backslash A$, lattice $E_{u}(A)$ of constraints u-expressible based on $A$ and lattice $E_{e}\left(A^{c}\right)$ of constraints e-expressible based on $A^{c}$ are dually isomorphic to each other, as may be shown through an argument grounded on Propositions 1.(ii) and 3.(ii).

As an example, let us consider the following set of binary 5 -tuples, each to be read as an assignment in context $(W, O)$ with $W=\left\{w_{1}, w_{2}, w_{3}, w_{4}, w_{5}\right\}$ and $O=\{0,1\}$ :

$$
\begin{aligned}
A= & \{00000,00010,00100,00110,01000,01010,01011,01100,01110,01111, \\
& 10000,10010,10011,10100,10110,10111,11001,11011,11101,11111\} .
\end{aligned}
$$

Detailed examination shows that the lattices of sets of variables that prove to be e-closed and, respectively, u-closed under constraint $A$ are the following collections:

$$
\begin{aligned}
\mathcal{E}_{A} & =\left\{\left\{w_{3}\right\},\left\{w_{1}, w_{3}\right\},\left\{w_{2}, w_{3}\right\},\left\{w_{3}, w_{4}\right\}, W\right\} \\
\mathcal{U}_{A} & =\left\{\left\{w_{3}\right\},\left\{w_{1}, w_{3}\right\},\left\{w_{2}, w_{3}\right\},\left\{w_{3}, w_{4}\right\},\left\{w_{3}, w_{5}\right\},\left\{w_{1}, w_{3}, w_{4}\right\},\left\{w_{2}, w_{3}, w_{4}\right\}, W\right\} .
\end{aligned}
$$

Thus, there are five e-expressible constraints and eight $\mathrm{u}$-expressible constraints on the basis of $A$. When partially ordered by set inclusion, both collections of constraints form a structure the diagram of which is shown in Figure 1 (note that initial constraint $A$ is, at the same time, the minimum in upper lattice $E_{e}(A)$ and the maximum in lower lattice $E_{u}(A)$ ).

\section{THE EXPRESSIVE POWER OF MULTIPLE PROJECTIONS}

Having discussed constraints which may be expressed on the basis of a given constraint through one single projection, we now enlarge the scope of analysis by considering the general case, in which the route leading from a given constraint to a new constraint consists of a chain of successive projections, of existential and/or universal type.

First, let us draw the syntactic profile of the problem, presuming that an alphabet is given consisting of a c-letter $A$ (to be used as the symbol for a constraint), a set $\mathcal{W}$ of v-letters (symbols for sets of variables), and symbols $\uparrow$ and $\downarrow$ for existential and universal projections. Then the concept of a $p$-formula (formula of projections) may be introduced by the following inductive definition: (i) c-letter $A$ is a p-formula; (ii) if $f$ is a p-formula and $U \in \mathcal{W}$, then $f \uparrow U$ and $f \downarrow U$ are p-formulas; (iii) any sequence of symbols is a p-formula only if it can be produced through a finite series of applications of rules (i) and (ii). This being the definition, any p-formula is a sequence of symbols of the following general aspect:

$$
A \rho_{1} U_{1} \cdots \rho_{m} U_{m}
$$




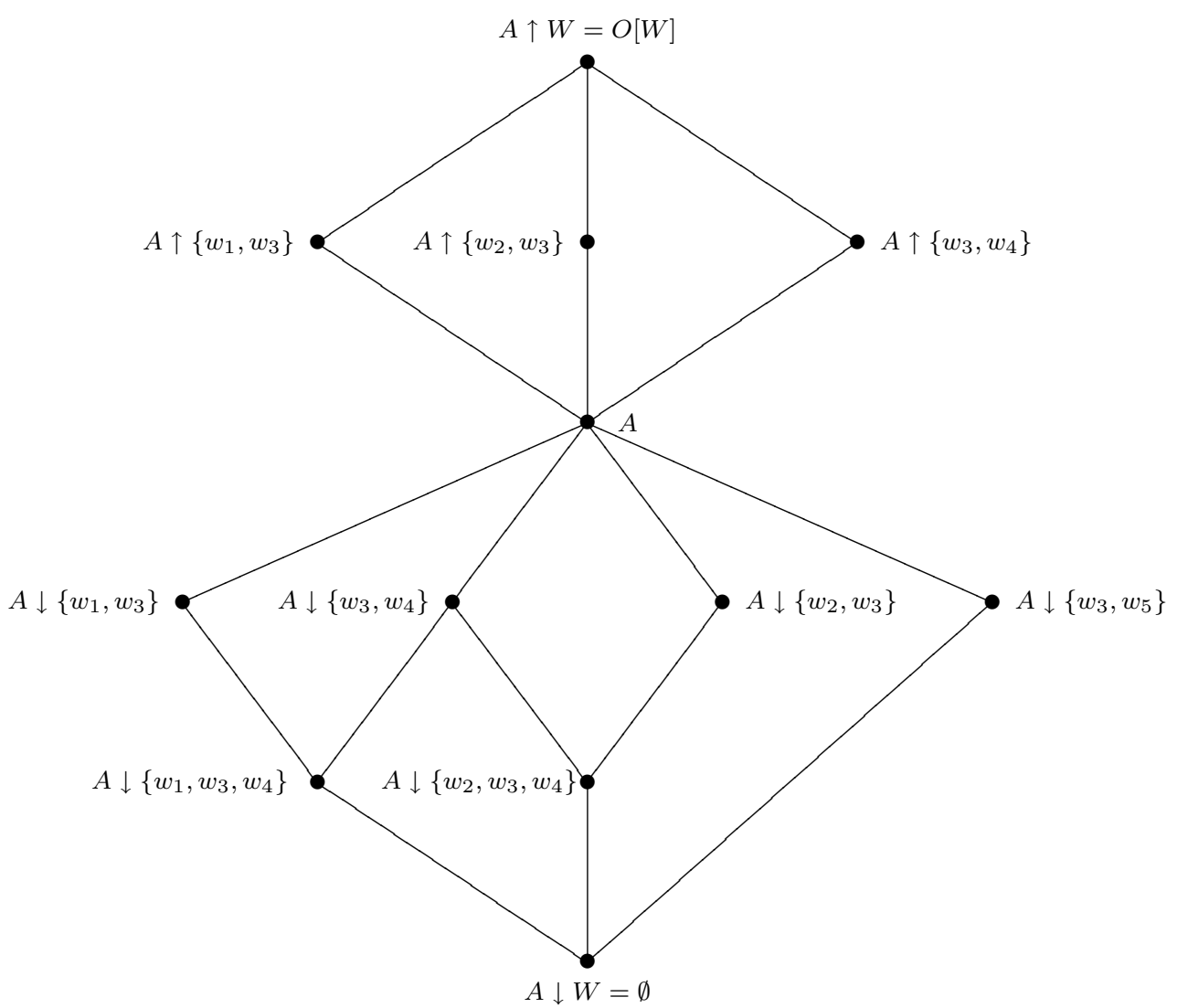

FIGURE 1. Hasse diagram of the double lattice of constraints that are e- or u-expressible based on constraint (11).

term $A$ (the root of the p-formula) is the presumed c-letter, terms $U_{1}, \ldots, U_{m}$ (some of which may be identical) are v-letters, term $\rho_{i}$ (for $i=1, \ldots, m$ ) is either $\uparrow$ or $\downarrow$, and number $m$ is called the length of the p-formula. The class of all p-formulas (rooted on $A$ ) is of infinite cardinality, since no upper bound is set to their (finite) length.

Shifting our perspective from the syntactic to the semantic side of the problem, let us assume that letters in the alphabet are given definite interpretations, in that a context $(W, O)$ is specified, c-letter $A$ is used to signify a selected subset of $O[W]$, and v-letters in $\mathcal{W}$ are used as labels for (all) subsets of set $W$ of variables in the context. Then, associated with any p-formula $A \rho_{1} U_{1} \cdots \rho_{m} U_{m}$, a sequence $\left(B_{0}, B_{1}, \ldots, B_{m}\right)$ of constraints becomes determined, which is recursively produced as follows:

$$
\begin{aligned}
& B_{0}=A, \\
& B_{i}=B_{i-1} \uparrow U_{i} \text { or } B_{i}=B_{i-1} \downarrow U_{i} \\
& \text { depending on whether } \rho_{i}=\uparrow \text { or } \rho_{i}=\downarrow, \text { for } i=1, \ldots, m .
\end{aligned}
$$

In this equation, $U_{1}, \ldots, U_{m}$ are subsets of $W, B_{0}, B_{1}, \ldots, B_{m}$ are subsets of $O[W]$, $\uparrow$ and $\downarrow$ are the set-theoretic operations of e- and u-projections, as defined by (2) and (3). In particular, the last constraint $B_{m}$ in the sequence is the result of multiple projections as described by the given p-formula, and is said to be expressed by that p-formula. Symbol $E_{p}(A)$ denotes the class of all constraints that are $p$-expressible 
on the basis of $A$, i.e., each of them is expressed by at least one p-formula rooted on $A$.

To study the inner structure of class $E_{p}(A)$, it is profitable to call into play pformulas of a special kind, which we call "normal" p-formulas. They constitute a set of formulas which is homogeneous and of finite cardinality but at the same time semantically exhaustive, in that any p-expressible constraint is expressed by some such formula (this statement is Proposition 7, which will be proved in the sequel). The special formulas to be defined are of two general types, which we call type $I$ and type II p-formulas and which are characterised by the following alternating schemes:

type I: $A \uparrow U_{1} \downarrow U_{2} \uparrow \cdots \uparrow U_{m}$ or $A \uparrow U_{1} \downarrow U_{2} \uparrow \cdots \downarrow U_{m}$ depending on whether $m$ is an odd or even number; type II: $A \downarrow U_{1} \uparrow U_{2} \downarrow \cdots \downarrow U_{m}$ or $A \downarrow U_{1} \uparrow U_{2} \downarrow \cdots \uparrow U_{m}$ depending on whether $m$ is an odd or even number.

As for any p-formula, once terms $A, U_{1}, \ldots, U_{m}$ are given definite meanings as a constraint and $m$ sets of variables, a definite sequence of constraints $\left(B_{0}=\right.$ $\left.A, B_{1}, \ldots, B_{m}\right)$ becomes associated with a type I or type II p-formula. Note that, for both special types, the general recursive rule (12) specialises as follows:

type I: $B_{i}=B_{i-1} \uparrow U_{i}$ or $=B_{i-1} \downarrow U_{i}$ depending on whether $i$ is odd or even;

type II: $B_{i}=B_{i-1} \downarrow U_{i}$ or $=B_{i-1} \uparrow U_{i}$ depending on whether $i$ is odd or even.

Based on these premises, the definition of the concept "normal p-formula" is as follows. A type I (resp., a type II) p-formula is a normal p-formula if, for each $i=1, \ldots, m$, set of variables $U_{i}$ is different from the largest free set of variables $F\left(B_{i-1}\right)$ and is an e-closed or a u-closed (resp., a u-closed or an e-closed) set of variables under $B_{i-1}$ depending on whether $i$ is an odd or even number. Note that this definition is not purely syntactic in its conception. This is because, in qualifying the concept, reference is made to the properties of being the largest free set and of being an e- or u-closed set of variables under a certain constraint, which are properties of a semantic nature and are well defined only if terms $A, U_{1}, \ldots, U_{m}$ are given as appropriate sets, not merely as letters of an alphabet. Also consider that, among normal p-formulas, as extreme examples in the category, we count constraint $A$ taken in isolation, any formula $A \uparrow U$ with $U \in \mathcal{E}_{A} \backslash\{F(A)\}$ and any formula $A \downarrow U$ with $U \in \mathcal{U}_{A} \backslash\{F(A)\}$; the first is a normal p-formula with no projection (its length is 0 ), the other two are normal p-formulas with one single projection (their length is 1).

There are some properties which can easily be derived from the definition of normal p-formulas and some previous results of our analysis. For one property, note that if $A \rho_{1} U_{1} \cdots \rho_{m} U_{m}$ is a normal p-formula, then the sets of variables involved in its construction form a strictly increasing sequence, i.e.:

$$
U_{0} \subset U_{1} \subset \ldots \subset U_{m}
$$

where $U_{0}=F(A)$. This is because the largest free set of variables $F\left(B_{i}\right)$ (for $i=0, \ldots, m-1)$ is the minimum in both lattice $\mathcal{E}_{B_{i}}$ of e-closed and lattice $\mathcal{U}_{B_{i}}$ of 
u-closed sets of variables (last paragraph of Section 3). Property (13) implies that there is an upper bound to the length of normal p-formulas, which is the cardinality of set $W$ of variables in the context (in this study, it is presumed to be finite). In turn, this fact implies that the number of normal p-formulas rooted on any fixed constraint is finite. Normal p-formulas which cannot be made longer by adding one or more projections on the right-hand side — while preserving the normality condition — are said to be maximal normal p-formulas.

Another simple result concerns the sequence $\left(B_{0}=A, B_{1}, \ldots, B_{m}\right)$ of constraints associated with a normal p-formula $A \rho_{1} U_{1} \cdots \rho_{m} U_{m}$, and is expressed by the following pair of statements:

if the formula is of type I, then $B_{0} \subset B_{1} \supset B_{2} \subset B_{3} \supset \ldots$ if the formula is of type II, then $B_{0} \supset B_{1} \subset B_{2} \supset B_{3} \subset \ldots$;

they are easily deduced from (4) and Proposition 5. The following proposition gives more results concerning this topic.

PRoposition 6. Let $A \rho_{1} U_{1} \cdots \rho_{m} U_{m}$ be a normal $p$-formula and $\left(B_{0}, B_{1}, \ldots, B_{m}\right)$ the associated sequence of constraints. These constraints are all different from one another (i.e., $B_{i} \neq B_{j}$ for all $0 \leq i<j \leq m$ ) and, if the formula is a maximal normal p-formula, then either $B_{m}=O[W]$ or $B_{m}=\emptyset$.

Proof. In the presumed conditions, let us refer to any two distinct positions $0 \leq i<$ $j \leq m$, the corresponding constraints $B_{i}$ and $B_{j}$, and set of variables $U_{i+1} \backslash U_{i}$ which, in view of (13), is a non-empty set. On one hand, we see that $U_{i} \subseteq F\left(B_{i}\right) \subset U_{i+1}$, due to Proposition 2.(iii) and the definition of a normal p-formula, so that $U_{i+1} \backslash U_{i}$ is not a subset of $F\left(B_{i}\right)$. On the other hand, $U_{i+1} \subseteq U_{j} \subseteq F\left(B_{j}\right)$, so that $U_{i+1} \backslash U_{i}$ is a subset of $F\left(B_{j}\right)$. Thus, $F\left(B_{i}\right) \neq F\left(B_{j}\right)$, which implies $B_{i} \neq B_{j}$. The second part in the proposition is simply due to the fact that $O[W]$ and $\emptyset$ are the only constraints such that the associated largest free set of variables is set $W$ of all variables in the context (see comments after equation (7)).

In its first part, the proven proposition ensures that all constraints in the sequence associated with any one normal p-formula are different from one another. Of course, this by no means implies that constraints in sequences associated with distinct normal p-formulas - whether rooted on the same or on different constraints - are all different from one another. It is quite possible for one and the same constraint to be expressed by different normal p-formulas, and the proposition signals this general fact in its second part: the largest constraint $O[W]$ and empty constraint $\emptyset$ are expressed by many different normal p-formulas, since any maximal normal p-formula expresses either the one or the other of these extreme constraints.

The following proposition asserts the semantic exhaustiveness of normal p-formulas: they constitute a special (finite) class, yet its expressive power equals the power of the (infinite) class of p-formulas in its entirety.

Proposition 7. Let $A, B \subseteq O[W]$ be any two constraints. If $B$ is p-expressible on the basis of $A$, then $B$ is expressed by some normal p-formula rooted on $A$. 
Proof. Let $A$ and $B$ be constraints so that $B$ is p-expressible on the basis of $A$. This means that there is some p-formula $A \rho_{1} U_{1} \cdots \rho_{m} U_{m}$ the result of which is $B$, i.e., the following set-theoretic equation is valid:

$$
B=A \rho_{1} U_{1} \cdots \rho_{m} U_{m}
$$

Our task is to prove that this p-formula can be transformed into another p-formula $A \sigma_{1} V_{1} \cdots \sigma_{n} V_{n}$, which is normal and has the same result, so that the following settheoretic equation is itself valid:

$$
B=A \sigma_{1} V_{1} \cdots \sigma_{n} V_{n}
$$

We prove this by showing how the given formula can be transformed into the new formula in a step-by-step manner. First stage of the proof: let us first consider the following set of indices:

$$
\left\{i: 1 \leq i \leq m, U_{i} \backslash F(A) \neq \emptyset\right\} .
$$

It may be the empty set, which would mean that each $U_{i}$ (for $\left.i=1, \ldots, m\right)$ is a free set under $A$, so that $B=A$ in view of (14), and we may conclude that $B$ is expressed by the normal p-formula with no projection and rooted on $A$. Then assume that (16) is a non-empty set, denote the smallest term in it by $t(0)$, and consider the following other set of indices:

$$
\begin{aligned}
& \left\{i: t(0)<i \leq m, \rho_{i} \neq \rho_{t(0)}, U_{i} \backslash \omega\left(U_{t(0)} \cup \cdots \cup U_{i-1}\right) \neq \emptyset\right\} \\
& \text { where } \omega=\epsilon_{A} \text { or }=v_{A} \text { depending on whether } \rho_{t(0)}=\uparrow \text { or }=\downarrow .
\end{aligned}
$$

Again, it may be that this set is empty, i.e., for all $t(0)<i \leq m$ either $\rho_{i}=\rho_{t(0)}$ or $U_{i} \subseteq \omega\left(U_{t(0)} \cup \cdots \cup U_{i-1}\right)$ (or both). In this case, by setting $\sigma_{1}=\rho_{t(0)}$ and $V_{1}=\omega\left(U_{t(0)} \cup \cdots \cup U_{m}\right)$, in view of (14) and due to Proposition 1.(iv) and the definitions of free and closed sets of variables, the following set-theoretic equation holds true:

$$
B=A \sigma_{1} V_{1}
$$

where $V_{1} \neq F(A)$, since $U_{t(0)} \subseteq V_{1}$ but $U_{t(0)} \backslash F(A) \neq \emptyset$. Thus we may say that target (15) has been attained with $n=1$, i.e., constraint $B$ is expressed by a normal p-formula with one single projection. Then assume that (17) is a non-empty set, so that there is the smallest term in it, which we denote by $t(1)$; put $\sigma_{1}=\rho_{t(0)}$ and $V_{1}=\omega\left(U_{t(0)} \cup \cdots \cup U_{t(1)-1}\right)$. For much the same reasons mentioned in the previous case, we see that p-formulas $A \sigma_{1} V_{1}$ and $A \rho_{1} U_{1} \cdots \rho_{t(1)-1} U_{t(1)-1}$ give the same result, so that, from (14) and through substitution, the following set-theoretic equation is derived:

$$
B=A \sigma_{1} V_{1} \rho_{t(1)} U_{t(1)} \cdots \rho_{m} U_{m}
$$

Note that, in the p-formula constituting the right-hand side of the equation, prefix $A \sigma_{1} V_{1}$ is a normal p-formula, and that $\sigma_{1} \neq \rho_{t(1)}$ and $U_{t(1)} \backslash V_{1} \neq \emptyset$, due to the conditions specifying set (17). Second stage of the proof: assume (as the inductive hypothesis) that a p-formula has been obtained so that the following set-theoretic equation holds true:

$$
B=A \sigma_{1} V_{1} \cdots \sigma_{r} V_{r} \rho_{t(r)} U_{t(r)} \cdots \rho_{m} U_{m}
$$


where prefix $A \sigma_{1} V_{1} \cdots \sigma_{r} V_{r}$ is a normal p-formula, $\sigma_{r} \neq \rho_{t(r)}$ and $U_{t(r)} \backslash V_{r} \neq \emptyset$. We must show how that p-formula can be transformed into another p-formula which is equivalent to it in result, and is such that the "normalised" prefix is one step longer, the "not yet normalised" suffix is at least one step shorter (possibly empty), and conditions accompanying equation (18) are preserved. For this aim, let us consider the following set of indices:

$$
\begin{aligned}
& \left\{i: t(r)<i \leq m, \rho_{i} \neq \rho_{t(r)}, U_{i} \backslash \omega\left(U_{t(r)} \cup \cdots \cup U_{i-1}\right) \neq \emptyset\right\} \\
& \text { where } \omega=\epsilon_{C} \text { or }=v_{C} \text { depending on whether } \rho_{t(r)}=\uparrow \text { or }=\downarrow \\
& \text { and } C=A \sigma_{1} V_{1} \cdots \sigma_{r} V_{r} .
\end{aligned}
$$

It may be the case that (19) is the empty set. Then, putting $\sigma_{r+1}=\rho_{t(r)}$ and $V_{r+1}=\omega\left(U_{t(r)} \cup \cdots \cup U_{m}\right)$, and for the same reasons mentioned above, we see that p-formulas $C \sigma_{r+1} V_{r+1}$ and $C \rho_{t(r)} U_{t(r)} \cdots \rho_{m} U_{m}$ are equivalent in result, so that the following set-theoretic equation is obtained from (18):

$$
B=A \sigma_{1} V_{1} \cdots \sigma_{r} V_{r} \sigma_{r+1} V_{r+1}
$$

where the right-hand side is a normal p-formula. In this case, we may say that target (15) has been attained with $n=r+1$. The alternative is that (19) is a non-empty set, so that there is its smallest term, which we denote by $t(r+1)$. In this case, if we put $\sigma_{r+1}=\rho_{t(r)}, V_{r+1}=\omega\left(U_{t(r)} \cup \cdots \cup U_{t(r+1)-1}\right)$ and adapt the above argument, we see that $C \sigma_{r+1} V_{r+1}$ and $C \rho_{t(r)} U_{t(r)} \cdots \rho_{t(r+1)-1} U_{t(r+1)-1}$ are p-formulas equivalent in their results, so that the following set-theoretic equation is derived from (18):

$$
B=A \sigma_{1} V_{1} \cdots \sigma_{r} V_{r} \sigma_{r+1} V_{r+1} \rho_{t(r+1)} U_{t(r+1)} \cdots \rho_{m} U_{m}
$$

Prefix $A \sigma_{1} V_{1} \cdots \sigma_{r} V_{r} \sigma_{r+1} V_{r+1}$ in the right-hand side of this equation is a normal p-formula and is one step longer than the prefix in the right-hand side of equation (18). Moreover, $\sigma_{r+1} \neq \rho_{t(r+1)}$ and $U_{t(r+1)} \backslash V_{r+1} \neq \emptyset$, as required. This process of "stepwise normalisation" of a p-formula may be repeatedly applied, and sooner or later it comes to a conclusion. Its final application corresponds to the first step in which the set of indices specified by (19) turns out to be the empty set.

We conclude this section with an example illustrating the process of construction of normal p-formulas (which is inductive and alternating in character) and the organization of the entire set of such formulas. Let $W=\left\{w_{1}, w_{2}, w_{3}, w_{4}\right\}$ be the set of variables, $O=\{0,1\}$ the set of values, and, relative to context $(W, O)$, let us introduce this constraint:

$$
A=\{0011,0100,0101,0110,0111,1011,1100,1101,1110,1111\} .
$$

Acting as for the example in the previous section, we find that the lattices of e-closed and $\mathrm{u}$-closed sets of variables under constraint $A$ are the following:

$$
\begin{aligned}
\mathcal{E}_{A} & =\left\{\left\{w_{1}\right\},\left\{w_{1}, w_{3}\right\},\left\{w_{1}, w_{4}\right\}, W\right\} \\
\mathcal{U}_{A} & =\left\{\left\{w_{1}\right\},\left\{w_{1}, w_{2}\right\},\left\{w_{1}, w_{3}, w_{4}\right\}, W\right\} .
\end{aligned}
$$


In particular, $F(A)=\left\{w_{1}\right\}$ is the largest set of variables free under $A$. In these conditions, we may construct 6 normal p-formulas of length 1; precisely, 3 formulas of type I (which are $A \uparrow\left\{w_{1}, w_{3}\right\}, A \uparrow\left\{w_{1}, w_{4}\right\}$, and $A \uparrow W$ ), and 3 formulas of type II $\left(A \downarrow\left\{w_{1}, w_{2}\right\}, A \downarrow\left\{w_{1}, w_{3}, w_{4}\right\}\right.$, and $\left.A \downarrow W\right)$. These formulas are pictured as nodes of the graph in Figure 2, setting the former in the upper part of the graph and the latter in the lower part, in accordance with relations $A \uparrow U \supseteq A \supseteq A \downarrow V$ (for all $U \in \mathcal{E}_{A}$ and $V \in \mathcal{U}_{A}$ ) which are ensured by (4).

Next, consider any one of the three normal p-formulas of type I and length 1 for example, formula $A \uparrow\left\{w_{1}, w_{3}\right\}$, which expresses the following constraint:

$\{0001,0011,0100,0101,0110,0111,1001,1011,1100,1101,1110,1111\}$.

We see that the lattice of u-closed sets of variables under this constraint is the following:

$$
\mathcal{U}_{A \uparrow\left\{w_{1}, w_{3}\right\}}=\left\{\left\{w_{1}, w_{3}\right\},\left\{w_{1}, w_{2}, w_{3}\right\},\left\{w_{1}, w_{3}, w_{4}\right\}, W\right\} .
$$

In particular, $\left\{w_{1}, w_{3}\right\}=F\left(A \uparrow\left\{w_{1}, w_{3}\right\}\right)$. Thus, there are 3 ways of developing formula $A \uparrow\left\{w_{1}, w_{3}\right\}$ into normal p-formulas of type I and length 2 ; these formulas are $A \uparrow\left\{w_{1}, w_{3}\right\} \downarrow\left\{w_{1}, w_{2}, w_{3}\right\}, A \uparrow\left\{w_{1}, w_{3}\right\} \downarrow\left\{w_{1}, w_{3}, w_{4}\right\}$, and $A \uparrow\left\{w_{1}, w_{3}\right\} \downarrow W$, which in Figure 2 are depicted as nodes at a lower level than node $A \uparrow\left\{w_{1}, w_{3}\right\}$, because the constraints they identify are subsets of constraint $A \uparrow\left\{w_{1}, w_{3}\right\}$. Instead, when we consider formula $A \uparrow W$, we find that it expresses the largest constraint $O[W]$ in the presumed context, and that the lattice of u-closed sets of variables under this constraint is $\mathcal{U}_{A \uparrow W}=\{W\}$. This means that $A \uparrow W$ is a maximal normal p-formula (of type I). It is depicted as a terminal node in the graph of Figure 2.

In a symmetric way, if we consider $A \downarrow\left\{w_{1}, w_{2}\right\}$ - which is one of the normal pformulas of type II and length 1 - we find that it expresses the following constraint:

$$
\{0011,0111,1011,1111\}
$$

and that the lattice of e-closed sets of variables under this constraint is as follows:

$$
\mathcal{E}_{A \downarrow\left\{w_{1}, w_{2}\right\}}=\left\{\left\{w_{1}, w_{2}\right\},\left\{w_{1}, w_{2}, w_{3}\right\},\left\{w_{1}, w_{2}, w_{4}\right\}, W\right\}
$$

with $\left\{w_{1}, w_{2}\right\}=F\left(A \downarrow\left\{w_{1}, w_{2}\right\}\right)$. This implies that $A \downarrow\left\{w_{1}, w_{2}\right\} \uparrow\left\{w_{1}, w_{2}, w_{3}\right\}$, $A \downarrow\left\{w_{1}, w_{2}\right\} \uparrow\left\{w_{1}, w_{2}, w_{4}\right\}$, and $A \downarrow\left\{w_{1}, w_{2}\right\} \uparrow W$ are the only possible expansions of $A \downarrow\left\{w_{1}, w_{2}\right\}$ into normal p-formulas of type II and length 2 . They are consistently depicted in Figure 2 as nodes at a level higher than node $A \downarrow\left\{w_{1}, w_{2}\right\}$. Instead, formula $A \downarrow W$ is a maximal normal p-formula of type II, and is given by a terminal node in Figure 2, because $\mathcal{E}_{A \downarrow W}=\{W\}$ is the lattice of e-closed sets of variables under the constraint it expresses, which is empty constraint $\emptyset$.

These rules may be applied to each normal p-formula $f$ obtained during the process. As seen, the main steps of each application are: (i) find the constraint $B$ expressed by formula $f$; (ii) find either lattice $\mathcal{U}_{B}$ or lattice $\mathcal{E}_{B}$, depending on whether the last projection sign in $f$ is $\uparrow$ or $\downarrow$; (iii) if set $W$ is the only member in the relevant lattice (i.e., $F(B)=W$ ), then declare $f$ a maximal normal p-formula; (iv) otherwise, expand $f$ into as many new p-formulas as there are sets $U \neq F(B)$ 


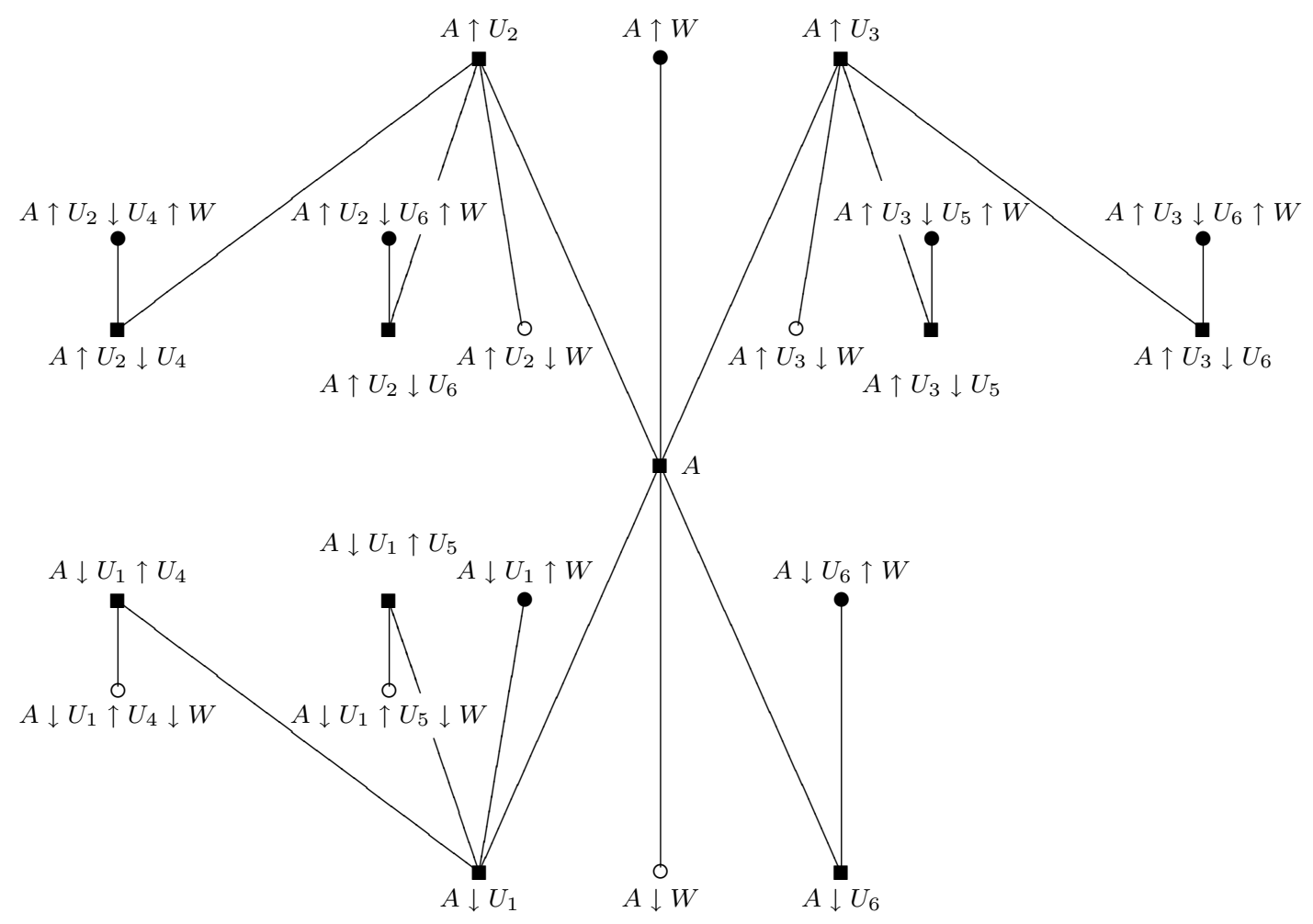

FIGURE 2. Normal p-formulas, of type I (upper part) and type II (lower part), rooted on constraint $A$ specified by (20). Subsets of variables: $U_{1}=\left\{w_{1}, w_{2}\right\}, U_{2}=\left\{w_{1}, w_{3}\right\}$, $U_{3}=\left\{w_{1}, w_{4}\right\}, U_{4}=\left\{w_{1}, w_{2}, w_{3}\right\}, U_{5}=\left\{w_{1}, w_{2}, w_{4}\right\}, U_{6}=\left\{w_{1}, w_{3}, w_{4}\right\}$. Filled and unfilled circles: maximal normal p-formulas which express the largest constraint $O[W]$ and, respectively, empty constraint $\emptyset$.

in the lattice, these formulas being $f \downarrow U$ or $f \uparrow U$ depending on whether the last projection sign in $f$ is $\uparrow$ or $\downarrow$. The complete set of normal p-formulas obtained by exhaustively applying this procedure within our example is that depicted in Figure 2. Note that, for ease of reading - and in accordance with the syntactic aspect of the process - we represent the whole structure as a tree, i.e., a connected acyclic graph. However, this structure is not a tree when it is viewed in the semantic (settheoretic) perspective, because different normal p-formulas may express the same constraint. For instance, formulas $A \downarrow\left\{w_{1}, w_{3}, w_{4}\right\}$ and $A \downarrow\left\{w_{1}, w_{3}\right\} \uparrow\left\{w_{1}, w_{3}, w_{4}\right\}$ both express the following constraint:

$$
\{0100,0101,0110,0111,1100,1101,1110,1111\} .
$$

Thus, there are cycles in the structure. Also note that, as ensured by Proposition 7, the set of $23 \mathrm{p}$-formulas in Figure 2 is semantically exhaustive, relative to root constraint $A$ specified by (20): any constraint which may be derived from $A$ through a (finite) sequence of projections is faithfully expressed by some of the $23 \mathrm{p}$-formulas in the Figure. Taking into account semantic equivalences between normal p-formulas, we see that distinct constraints p-expressible based on $A$ are 9 in number (among them, the largest constraint $O[W]$ and the empty constraint $\emptyset$, which correspond to the leaves of the tree). 


\section{THE COMBINED EXPRESSIVE POWER OF PROJECTIVE AND BOOLEAN OPERATIONS}

Before concluding, I wish to add a few notes concerning the expressive power of a composite system, which besides e-projection $\uparrow$ and u-projection $\downarrow$, also comprises complementation ${ }^{c}$, union $\cup$ and intersection $\cap$, the Boolean operations on constraints. There is a correspondence between the basic operators in this system and those in a first-order predicate calculus: projective operations correspond to quantifiers $\exists$ and $\forall$, and Boolean operations to connectives $\neg, \vee$ and $\wedge$.

The alphabet is $\left(\mathcal{A}, \mathcal{W}, \uparrow, \downarrow,^{c}, \cup, \cap,(),\right)$ in which, besides symbols for operations and brackets for syntactic use, we have a (finite) set $\mathcal{A}$ of c-letters (symbols for primitive constraints) and a (finite) set $\mathcal{W}$ of v-letters (symbols for sets of variables). Using symbols in this alphabet we may produce an infinite number of $p b$-formulas (formulas with projective and Boolean operations), the inductive definition of which is as follows: (i) for each $A \in \mathcal{A}$, symbol $A$ is a pb-formula; (ii) if $f$ is a pb-formula and $U \in \mathcal{W}$, then $(f) \uparrow U$ and $(f) \downarrow U$ are pb-formulas; (iii) if $f$ and $g$ are pbformulas, then $(f)^{c},(f) \cup(g)$ and $(f) \cap(g)$ are pb-formulas; (iv) any sequence of symbols taken from the alphabet is a pb-formula only if it can be produced through a finite series of applications of rules (i)-(iii). Those pb-formulas which do not contain any occurrence of symbols ${ }^{c}, \cup, \cap$ are the $p$-formulas (purely projective formulas) already considered in the previous section. Symmetrically, those pb-formulas which do not contain any occurrence of symbols $\uparrow, \downarrow$ are the $b$-formulas (purely Boolean formulas). All pb-formulas complying with scheme $(g) h$, where $g$ stands for a bformula and $(A) h$ for a p-formula ( $A$ is any c-letter), are called here normal $p b$ formulas. Moreover, assuming $\mathcal{A}=\left\{A_{1}, \ldots, A_{n}\right\}$, any b-formula complying with this scheme:

$$
\left(A_{1}^{s_{1,1}} \cap \cdots \cap A_{n}^{s_{1, n}}\right) \cup \cdots \cup\left(A_{1}^{s_{k, 1}} \cap \cdots \cap A_{n}^{s_{k, n}}\right)
$$

where, for $j=1, \ldots, n$ and $h=1, \ldots, k$, term $A_{j}^{s_{h, j}}$ is either $A_{j}$ or $A_{j}^{c}$, qualifies as a normal b-formula (such formulas correspond to the "full disjunctive normal forms", as they are defined in formal logic; cf. [Mendelson, 1964, §1.3]).

Now suppose that a context $(W, O)$ is specified, each c-letter $A \in \mathcal{A}$ is associated with a definite constraint in that context (i.e., a subset of $O[W]$ ), and each v-letter $U \in \mathcal{W}$ is associated with a definite set of variables (i.e., a subset of $W$ ). Constraints directly associated with c-letters in $\mathcal{A}$ qualify as the "primitive constraints" in the interpretation. In these conditions, each b-formula $f$ expresses a certain constraint in the given context: that constraint is the result we obtain by starting from the primitive constraints the symbols of which occur in formula $f$ and by applying the Boolean operations specified in the formula in an orderly manner. Denote by $E_{b}(\mathcal{A})$ the set of all constraints which are b-expressible on the basis of the chosen primitive constraints, i.e., are expressed by b-formulas in the presumed conditions. Similarly, denote by $E_{p b}(\mathcal{A})$ the set of all constraints which are $p b$-expressible in those conditions, i.e., they are expressed by pb-formulas starting from the accepted family of primitive constraints. We know from the general theory of Boolean algebras that $E_{b}(\mathcal{A})$ is a field of sets; more precisely, it is the smallest field of subsets of $O[W]$ including family $\mathcal{A}$ of primitive constraints. It is easily recognised that $E_{p b}(\mathcal{A})$ is 
itself a field of sets (a case of "Boolean algebra with operators", in the sense of [Jónsson, Tarski, 1951]) and that $E_{b}(\mathcal{A}) \subseteq E_{p b}(\mathcal{A})$.

By proving Proposition 7 we have established that for any p-formula there is a normal p-formula semantically equivalent to it, in that both express the same constraint (in the presumed interpretation). A similar rule holds true for b-formulas and is a well-known fact in formal logic: for any b-formula there is a normal b-formula semantically equivalent to it. In view of these two results, a question naturally arises: may we say that, for any pb-formula, there is a normal pb-formula semantically equivalent to it? Note that, if a pb-formula $f$ is semantically equivalent to a normal pb-formula $(g) h$, then by virtue of the above results it is also semantically equivalent to a pb-formula $\left(g^{\prime}\right) h^{\prime}$ such that $g^{\prime}$ is a normal b-formula and $(A) h^{\prime}$ a normal p-formula. This is a highly regular kind of pb-formula.

There is a passage in our analysis indicating that the above question cannot be positively answered in general. It corresponds to part (i) of Proposition 3, which states that order inversion between projective operations and union or intersection operations - i.e., the basic move for transforming any given pb-formula into a normal pb-formula semantically equivalent to it - is conditional upon certain freedom relationships between the sets of variables and the constraints involved. A simple example which confirms the negative expectation about the question is as follows. Assume $W=\left\{w_{1}, w_{2}\right\}$ and $O=\{0,1\}$ (so that $O[W]=\{00,01,10,11\}$, where $00=\left\{\left(w_{1}, 0\right),\left(w_{2}, 0\right)\right\}$, etc. $)$; then choose $A_{1}=\{00\}$ and $A_{2}=\{11\}$ as primitive constraints, and consider $B=\{00,10,11\}$. We see that there are pb-formulas capable of expressing $B$ based on $\left\{A_{1}, A_{2}\right\}$ (most simply, $B=\left(A_{1} \uparrow\left\{w_{1}\right\}\right) \cup\left(A_{2} \uparrow\left\{w_{2}\right\}\right)$ ). On the contrary, by examining the field of constraints generated by $\left\{A_{1}, A_{2}\right\}$ and possible projections of those constraints, we reach the conclusion that there is no normal pb-formula expressing $B$.

However, there is an easily recognisable kind of pb-formula for which the above question yields a positive answer in general. In stating the result (the next proposition), reference is made to the "scope" of any projective operation in a pb-formula, which means that part of the formula which lies inside the brackets closing immediately before the symbol of the operation (see rule (ii) in the above definition of pb-formulas).

PROPOSITION 8. If $f$ is a pb-formula to be interpreted with reference to a definite family of primitive constraints and so that for every projective operation in it, the set of variables specifying the operation is free under each of the primitive constraints occurring in the formula outside the scope of that operation, then there is a normal pb-formula $(g) h$ semantically equivalent to $f$.

Proof. Under the presumed freedom relationships between sets of variables and primitive constraints, pb-formula $f$ can be transformed into an equivalent normal pb-formula $(g) h$ in a step-by-step manner, thanks to equations in Propositions 1.(ii) and 3.(i) (and in view of Propositions 2.(iv) and 3.(ii)).

This result is the counterpart for constraints of a well-known fact in the theory of first-order predicate calculi: any formula in such a calculus has an equivalent "prenex 
normal form", i.e., an equivalent formula in which all quantifiers - if any - are lined up together to form a prefix in the formula (cf. [Mendelson, 1964, §2.10]).

\section{COMPARISONS AND CONCLUSIONS}

We have considered relational constraints, as they are defined in the theory of constraint networks, and two special operations - existential and universal projections - on constraints, forming a fragment of the set-theoretic algebra of constraints (other basic operations of this algebra have been mentioned in the previous section). In the Introduction, we pointed out a correspondence between both projective operations and the existential and universal quantifiers in a first-order predicate calculus, this correspondence being the reason for calling the two projective operations as we have proposed (other elementary correspondences between the algebra of constraints and the semantics of a predicate calculus have been mentioned in the previous section). But there are other theoretical constructions in pure and applied mathematics which have (partial) similarities with the system examined in our study. In this last section, I wish to mention a few of these theoretical constructions, some of which have proven to be of use for mathematical modelling in psychological science, and social sciences more generally. For these constructions, I point out elementary similarities and differences relative to the system examined here, thus specifying the peculiarities of my present contribution.

In order to define any relational constraint, we must presume that two separate fundamental sets are given: a set of variables $W$ and a set of values $O$. Reference to a pair - sometimes a triple - of separate fundamental sets is a feature which may be encountered in other significant theoretical constructions. For example, modelling in "formal concept analysis" (in its standard version) involves a set $O$ of "objects" and a set $A$ of "attributes"; a "context" $C$ is defined as a Boolean matrix having the rows indexed by terms in $O$ and columns by terms in $A$, i.e., a function $C: O \times A \rightarrow\{0,1\}$, where $C(o, a)=1$ means that object $o$ is endowed with attribute $a$, and $C(o, a)=0$ means the contrary, for any $o \in O$ and $a \in A$ (cf. [Ganter, Wille, 1999, §1.1]). One step higher in complexity, "many-valued contexts" are considered, involving a third set $V$ of "values"; a many-valued context $C$ is a matrix $O \times A$, the entries of which are values in $V$, i.e., a function $C: O \times A \rightarrow V$, in which, for any $o \in O$ and $a \in A$, value $C(o, a) \in V$ specifies the level reached by attribute $a$ in object $o$, or else the degree to which object $o$ possesses attribute $a$ (cf. [Ganter, Wille, 1999, §§1.3, 2.4; Belohlavek, 2000]). Abstractly similar is the concept of "interaction matrix" in the theory of "Chu-spaces": in this theory, basic sets corresponding to $O, A$, and $V$ are called the "carrier" (set of points), "co-carrier" (set of states), and "alphabet" (set of values), and an interaction matrix is defined as a function from $O \times A$ into $V$ (cf. $[\text { Pratt, 1999] })^{3}$. But there are conspicuous peculiarities of the theory of relational constraints, as compared with these other theoretical paradigms. As a rule, any specific constraint $A$ in a context $(W, O)$ involves a selected subset $U$ of whole set

\footnotetext{
${ }^{3}$ I thank one of the anonymous referees for calling my attention on this very wide and flexible theoretical paradigm, recently developed within the framework of the theory of categories.
} 
$W$ of variables ( $U$ qualifies as the "scope" of $A$ ), and is defined as a set of functions (value assignments) having $U$ as the domain and $O$ as the codomain. The structure associated with a "constraint satisfaction problem" is a "constraint network", i.e., a family of local constraints thus defined, the scopes of which may differ from one another. Once a constraint network is defined, the principal aim of the procedures studied in the theory is to find a "solution" to the network, i.e., a value assignment to all variables in $W$ so that all constraints in the network are satisfied (i.e., for each constraint in the network, the e-projection of the global assignment relative to the scope of the constraint is a local assignment belonging to the constraint). Quite different, for example, is the principal aim of formal concept analysis: given a Boolean context $C: O \times A \rightarrow\{0,1\}$, it brings to light a structure inherent in both basic sets $O$ and $A$, which expresses itself as a pair of dually isomorphic lattices of subsets, i.e., the "lattice of extents" on $O$ and the "lattice of intents" on $A$.

Regarding constraints, we have examined two special operations - existential and universal projections - which are dually related to each other and constitute a fragment of the set-theoretic calculus on constraints. Also for these two concepts we can find similar constructs in other domains of mathematics. Examples are "cylindrification" $c_{i}$ as an essential component of "cylindric algebras", and mapping $C_{\Gamma}$ as a basic operator in "polyadic algebras", both of which resemble existential projection in their general meanings and properties (cf. [Németi, 1991; Monk, 2000]). These correspondences are not surprising, when we consider that existential projection - as specified by equation (2) — is related to existential quantification in a firstorder predicate calculus, and that both cylindric algebras and polyadic algebras are salient results of the program of "algebraization of logic", as first-order predicate calculi (with and without equality) are specifically concerned (cf. [Andréka, Németi, Sain, 2001]). Other meaningful correspondences are found in the basic concepts of "descriptive set theory", specifically the concepts of "projection" $\exists^{\mathcal{Y}} P$ and "dual projection" $\forall^{\mathcal{Y}} P$ of a "pointset" $P$ along a "product space" $\mathcal{Y}$ (cf. [Moschovakis, $1980, \S 1 \mathrm{C}])^{4}$ : "pointsets", as defined in the theory, bear a generic resemblance to "constraints" in our study, in that both are subsets of multidimensional sets, and projection $\exists^{\mathcal{Y}}$ and dual projection $\forall^{\mathcal{Y}}$ are analogous to existential and universal projections in their general meanings ${ }^{5}$. Now, the approach adopted in our analysis is similar to that in descriptive set theory, in that it explicitly considers both projections of the existential type and projections of the universal type (in the theories of cylindric and polyadic algebras, only operators of the existential type are directly considered, since those of the universal type are derivable from them by means of negation, due to the equivalence of $(\forall x)(p(x))$ and $\neg(\exists x)(\neg p(x))$ in predicate logic). Our approach is also similar to that in the theory of polyadic algebras, in that projections of constraints are taken in relation to arbitrary subsets of set $W$ of variables ${ }^{6}$,

\footnotetext{
${ }^{4}$ Again, I thank the same referee for pointing out these correspondences. Descriptive set theory arose early in the twentieth century when dealing with "definability problems" for subsets of multidimensional geometric spaces (descriptive set theory as "definability theory for the continuum"; cf. [Moschovakis, 1980, p. 11]).

${ }^{5}$ In the historical perspective, it is worth noting that there are intersections between the roots of algebraic logic for predicate calculi and the roots of descriptive set theory (for example, [Kuratowski, Tarski,1931]).

${ }^{6}$ More precisely, this is a trait of the traditional way of presenting the theory. For references,
} 
which is homogeneous in its conception (cylindrification in a cylindric algebra is an operation involving one single variable at each application, whereas projections and dual projections in descriptive set theory may involve spaces of different kinds, like set $\omega$ of natural numbers, or any specified product space $\mathcal{Y}$ ).

We have discussed existential and universal projections of constraints with the specific purpose of exploring the "expressive power" of these operations, i.e., establishing which constraints can be produced by starting from some "input constraint" and applying the projective operations one or more times. In a general perspective, this is an example of the kind of problems characterizing "definability theory": given a selected set of primitive terms identifying objects of a certain kind, and given a system of rules for combining those terms into meaningful expressions, find the set of all objects that can be identified by those expressions, regardless of their syntactic length and complexity (cf. [Addison, 2004]). Definability problems so conceived are encountered in several specific parts of mathematics, as may be expected, since they ultimately concern the relation between the syntactic and semantic sides of a mathematical theory - primitive terms and combined expressions pertain to syntax, initial objects and definable objects to semantics. Confining ourselves to considering a couple of examples of direct concern for the psychological and social sciences, we mention here the "definability criterion of meaningfulness" in measurement theory (numerical expressions are judged to be meaningful relative to a measurement scale if they can be formed with basic terms in the numerical system qualifying that scale; cf. [Weitzenhoffer, 1951; Narens, 1988, 2002; Dzhafarov, 1995]) and the completeness condition of an algebraic social network (given some basic binary relations on a domain, the network is the set of all binary relations which are constructible, i.e., definable based on them through repeated composition; cf. [Boyd, 1991; Pattison, 1993]). The definability problem is also a core problem in descriptive set theory, and there is a generic resemblance between the perspective of analysis in this paper and the aspect of the problem in that theory, due to the above-stated correspondence between existential and universal projections in our conception, and projection and dual projection in descriptive set theory (in particular, cf. [Moschovakis, 1980, §8B], as regards "elementary definability" or "first-order definability" in a structure of several relations). Besides referring specifically to existential and universal projections of constraints on a finite context, our treatment of the definability problem distinguishes itself in two respects. One is that we explore the organization of the set of constraints definable through one projection (of existential or universal type); more precisely, the organization of the set of constraints which may be derived from a fixed input constraint by varying the subset of variables involved in the projection. By proving Proposition 5, we have shown that sets of e-expressible and, respectively, $\mathrm{u}$-expressible constraints, when internally ordered by set inclusion, are lattices of constraints, the one isomorphic to the lattice of sets of variables being e-closed under the input constraint, the other dually isomorphic to the lattice of sets of variables being u-closed under that constraint. The other respect is that we search for a small variety of regular formulas that can fully represent the whole variety of constraints definable through arbitrary sequences of projections from any fixed input constraint. By proving Proposition 7, we have shown that the set of normal p-formulas - which

cf. [Németi, 1991, p. 526]. 
is finite when the number of variables is finite - is a set with the required properties, so that it allows for efficient control over the whole set of p-expressible constraints.

\section{REFERENCES}

ADDISON J.W., "Tarski's theory of definability: common themes in descriptive set theory, recursive function theory, classical pure logic, and finite-universe logic", Annals of Pure and Applied Logic 126, 2004, p. 77-92.

ANDRÉKA H., NÉMETi I., SAIN I., "Algebraic logic", D.M. Gabbay, F. Guenthner (eds), Handbook of philosophical logic, Volume 2, Dordrecht, Kluwer Academic Publishers, 2001, p. 133-247.

BELOHLAVEK R., "Fuzzy Galois connections and fuzzy concept lattices: from binary relations to conceptual structures", V. Novak, I. Perfilieva (eds), Discovering the world with fuzzy logic, Heidelberg, Physika Verlag, 2000, p. 462-494.

BIRKhoff G., Lattice theory, Providence (RI), American Mathematical Society, 1967.

BOYD J.P., Social semigroups. A unified theory of scaling and blockmodelling as applied to social networks, Fairfax (VA), George Mason University Press, 1991.

DEChter R., Constraint processing, San Mateo (CA), Morgan Kaufmann, 2003.

DZHAFAROV E.N., "Empirical meaningfulness, measurement-dependent constants, and dimensional analysis", R.D. Luce, M. D’Zmura, D.D. Hoffman, G. Iverson, K. Romney (eds), Geometric representations of perceptual phenomena, Hillsdale (NJ), Erlbaum, 1995, p. 113134.

GANTER B., WILle R., Formal concept analysis: Mathematical foundations, Berlin, Springer, 1999.

Halmos P., Lectures on Boolean algebras, London, Van Nostrand, 1963.

JEAVONS P., COHEN D., GYSSENS M., "How to determine the expressive power of constraints", Constraints 4, 1999, p. 113-131.

JÓnsson B., TARSKi A., "Boolean algebras with operators. Part I", American Journal of Mathematics 73, 1951, p. 891-939.

KURATOWSKI C., TARSKI A., "Les opérations logiques et les ensembles projectifs", Fundamenta Mathematicae 17, 1931, p. 240-248.

MAIER D., The theory of relational databases, Rockville (MD), Computer Science Press, 1983.

MENDELSON E., Introduction to mathematical logic, Princeton (NJ), Van Nostrand, 1964.

MONK J.D., "An introduction to cylindric set algebras", Logic Journal of IGPL 8, 2000, p. $451-496$.

MONTANARI U., "Networks of constraints: fundamental properties and applications to picture processing", Information Sciences 7, 1974, p. 95-132.

moschovakis Y.n., Descriptive set theory, Amsterdam, North-Holland, 1980.

NAREns L., "Meaningfulness and the Erlanger program of Felix Klein", Mathématiques Informatique et Sciences humaines 101, 1988, p. 61-71.

NAREns L., Theories of meaningfulness, Mahwah (NJ), Erlbaum, 2002.

NÉMETI I., "Algebraization of quantifier logics, an introductory overview", Studia Logica 50, 1991, 485-569. 
PATTISOn P.E., Algebraic models for social networks, New York, Cambridge University Press, 1993.

PRATT V., "Chu spaces from the representational viewpoint", Annals of Pure and Applied Logic 96, 1999, p. 319-333.

WEITZENHOFFER A., "Mathematical structures and psychological measurement", Psychometrika 16, 1951, p. 387-406. 